\title{
ESTUDIOS
}

\section{[EL AVANCE DE LA URBANIZACIÓN DEL CAMPO EN LA REGIÓN METROPOLITANA DE CHILE Y SUS EFECTOS ESPACIALES]}

\section{Gladys Armijo Z.}

Departamento de Geografía

Facultad de Arquitectura y Urbanismo de la Universidad de Chile.

Héctor Caviedes B.

Departamento de Ciencias Históricas

Facultad de Filosofía y Humanidades de la Universidad de Chile.

\section{RESUMEN}

En este trabajo, se presentan los resultados de investigaciones que han sido llevadas a cabo por los autores del presente artículo, desde el año 1994, en el área Sur de la Región Metropolitana (Comunas de San Bernardo, Pirque y Paine).

En virtud de lo anterior, se observa un acelerado proceso de urbanización del campo que conlleva en sí la desaparición de las formas de vida características y propias del ámbito rural. Este proceso (en términos de calidad de vida) se manifiesta a través de una sub-urbanización y otra, de elite. Ambas son funcionales a la modernización agraria y se presentan en forma contradictoria.

\section{La metrópolis de Santiago (Fig. № 1)}

Una de las características más notables del proceso de asentamiento humano en América Latina y el Caribe ha sido la tendencia cada vez mayor a la urbanización. Esto conllevó obviamente, a una aguda concentración de la población, generando nuevos sistemas urbanos al interior de cada país e incluso en las regiones.

Así surge el fenómeno de la ciudad primada, representada en la mayoría de los casos por las capitales latinoamericanas. En función de este proceso, Santiago, al igual que el resto de las capitales de nuestro sub-continente, llegó a concentrar un altísimo porcentaje de la población total del país (30 al 40\%), y al mismo tiempo, aglutinó a la mayor parte de la infraestructura industrial, comercial y financiera. En consecuencia, se puede afirmar que la primacía urbana fue la expresión de una notable concentración de actividades productivas y de población y por ende, causal de los agudos desequilibrios regionales internos del país. Este proceso de ciudad primada, comenzó a presentarse a partir de los años cuarenta (TABLA No 1).

El crecimiento explosivo de Santiago, a partir de los años cuarenta tiene su 
origen en las migraciones campo-ciudad. El éxodo del campo alcanzó su fase cúlmine entre los años 40 y 60, lo que llegó a explicar el 52,7\% del crecimiento de Santiago para el período intercensal 1940-1952 y del 38,1\% para los años 1952-1960 (Hurtado, C. 1996).

Las citadas migraciones contribuyeron a agudizar los problemas de desempleo, déficit de vivienda e infraestructura, como también de equipamiento y servicios básicos de la ciudad en general. La expresión espacial, en cuanto a la urbanización, fue una aguda segregación socioeconómica.

En relación a la expansión física de la ciudad, fue evidente que ella se dirigiera hacia las áreas rurales circundantes, así por ejemplo, el área urbana de nuestra ciudad capital se ha cuadruplicado en los últimos 50 años, significando sólo para el período de 1970-1991 la pérdida de 16.699 hectáreas de suelos agrícolas, lo que es superior a la área ocupada por Santiago en 400 años, ya que ella tenía, hacia 1940, 11.348 has. (CIREN-CORFO, 1992).

CRECIMIENTO POBLACIONAL
\begin{tabular}{|l|l|l|l|l|}
\multicolumn{7}{c|}{ Y EXPANSION FISICA DEL SANTIAGO } \\
\hline \multirow{2}{*}{$\begin{array}{l}\text { AÑOS } \\
\end{array}$} & $\begin{array}{l}\text { POBLACION } \\
\text { DE } \\
\text { SANTIAGO }\end{array}$ & $\begin{array}{l}\text { POBLACION } \\
\text { TOTAL DEL } \\
\text { PAIS }\end{array}$ & $\begin{array}{l}\text { SUPERFICIE } \\
\text { (hectáreas) }\end{array}$ & $\begin{array}{l}\text { DENSIDAD } \\
\text { (hab./hás.) }\end{array}$ \\
\hline & & & & \\
\hline 1907 & 332.724 & 3.231 .022 & --- & --- \\
\hline 1920 & 507.296 & 3.730 .235 & --- & --- \\
\hline 1930 & 696.231 & 4.287 .445 & --- & --- \\
\hline 1940 & 952.075 & 5.023 .539 & 11.348 & 84.0 \\
\hline 1952 & 1.353 .400 & 5.932 .995 & 15.570 & 86.9 \\
\hline 1960 & 1.907 .378 & 7.641 .115 & 22.880 & 83.4 \\
\hline 1970 & 2.436 .398 & 8.884 .769 & 29.480 & 94.3 \\
\hline 1982 & 3.650 .541 & 11.275 .440 & 38.364 & 103.6 \\
\hline 1992 & 4.679 .900 & 13.348 .401 & 46.179 & 101.3 \\
\hline
\end{tabular}

Fuente: INE Censos de Población y Plan Regulador Metropolitano de Santiago 1992.

En este proceso de expansión física de la metrópolis de Santiago hacia sus bordes rurales, los suelos agrícolas comenzaron a experimentar una activa competencia de uso, siendo el rural desplazado, perdiendo en definitiva éstos, su carácter agrícola en beneficio del área edificada. En muchos casos este proceso fue precedido por un período de "anticipación urbana", mediante el cual el uso agrícola lograba permanecer en forma residual en espera que los precios 
tendieran a ser más ventajoso para su venta, es decir, para construcciones urbanas.

Los actores sociales que se involucraron, en forma directa, en este proceso estuvieron relacionados con agentes públicos y privados que elaboraron programas de vivienda básica y también, inmobiliarias que comenzaron a activar el mercado del suelo agrícola para construir futuras urbanizaciones. Esto implicó que estos suelos se vieran sometidos a mayores especulaciones comerciales.

La información estadística del último lustro revela que el ritmo de crecimiento del gran Santiago tiende a atenuarse, alcanzando valores similares al crecimiento vegetativo (1,7\% anual), debido a un cambio en el destino de las migraciones campo-ciudad, es decir, éstas se dirigen a nuevos focos de atracción de población que estarían representados por la ciudades intermedias. De esta manera en la Región Metropolitana, las ciudades menores están creciendo a un ritmo mucho más acelerado que el Gran Santiago (Fig. № 3), lo que conlleva a una alteración del sistema urbano regional metropolitano.

TABLA N²

"Crecimiento poblacional de las ciudades de la Región Metropolitana

1982-1992"

\begin{tabular}{|l|l|l|l|}
\hline CIUDADES & $\mathbf{1 9 8 2}$ & $\mathbf{1 9 9 2}$ & $\begin{array}{l}\text { CRECIMIENTO } \\
\text { INTERCENSAL } \\
(\%)\end{array}$ \\
\hline Batuco & 3.870 & 7.386 & 90.85 \\
\hline Buin & 18.071 & 33.059 & 82.94 \\
\hline Colina & 15.504 & 33.459 & 115.81 \\
\hline Curacaví & 7.877 & 11.866 & 50.64 \\
\hline El Monte & 13.334 & 17.843 & 33.82 \\
\hline Esmeralda & 2.873 & 3.697 & 26.68 \\
\hline Isla de Maipo & 6.667 & 9.731 & 45.96 \\
\hline Melipilla & 33.684 & 45.722 & 35.74 \\
\hline Padre Hurtado & 10.060 & 25.783 & 42.76 \\
\hline Paine & 7.346 & 12.818 & 74.49 \\
\hline Peñaflor & 36.497 & 46.711 & 27.99 \\
\hline Puente Alto & 110.153 & 254.127 & 130.7 \\
\hline San Bernardo & 117.187 & 179.396 & 53.09 \\
\hline San José de Maipo & 4.429 & 5.307 & 19.82 \\
\hline
\end{tabular}




\begin{tabular}{|l|l|l|l|}
\hline Talagante & 24.884 & 37.198 & 49.49 \\
\hline Santiago & 3.654 .760 & 4.295 .593 & 17.53 \\
\hline
\end{tabular}

Fuente : Censos de Población 1982 y 1992 INE

Cabe destacar que estos cambios que se están produciendo en la Región Metropolitana de Chile son paralelos a los que están observando en el resto de los países latinoamericanos. En las últimas décadas se ha consolidado el carácter urbano de la región.

En 1995, el 73,4\% de su población (351 millones de personas) vivía en áreas urbanas. Este nivel de urbanización ponía a América Latina y el Caribe a la par con regiones como Europa y a poca distancia de Estados Unidos y el Japón. Esta tendencia urbanizadora ha combinado la intensificación de la ocupación territorial con un aumento del número de localidades urbanas. Las informaciones censales muestran un tendencia, más o menos fuerte según los países, hacia una menor concentración demográfica en la ciudad principal (CEPAL, 1995).

Los nuevos patrones de urbanización que se observan en la Región Metropolitana de Chile, al igual que en el resto de los países latinoamericanos, se desarrollan en el contexto de la globalidad, con el consiguiente entrelazamiento de los procesos globales, nacionales y locales. Esta situación origina gravitaciones diferenciales en la construcción de los espacios geográficos. En este aspecto, Uribe señala..." ( )... el proceso de globalización destruye distancias y abate los tiempos, junto a un proceso simultáneo de fragmentación que secciona en múltiple pedazos a la población, a sus territorios y a sus culturas. Las discusiones e interpretaciones acerca de esta situación inédita se producen en un momento histórico en que se habla de la Sociedad Post-Industrial, Sociedad Informática, Sociedad de Servicios, Sociedad Tecnológica o Sociedad Post-Modernista".

Latinoamérica como región urbana respondería, entonces, a la noción de la "aldea global" del nuevo sistema-mundo. En su interior, la metrópolis de Santiago surge como un eje financiero entroncado a la estructura mundial de la industria de las finanzas. Todo lo anterior presupone una profunda reorganización del territorio metropolitano.

Sin embargo, pese al predominio de la población urbana en América Latina y el Caribe, el volumen de la población rural, 127 millones de personas ilustra por sí solo la importancia que aún tiene el mundo rural. Si a lo anterior se añaden las condiciones de vida más precarias que, en promedio, enfrenta la gente del campo, así como el atraso tecnológico de la agricultura campesina, se puede decir que tanto en términos cuantitativos como cualitativos, los asentamientos y la población dispersa en zonas rurales todavía constituyen objetivos prioritarios para el desarrollo (CEPAL, 1995). 


\section{La modernización del campo chileno}

Entre los años 1973-75 se define la estrategia política-económica que se impone en el país. La adopción del modelo neo-liberal implicó grandes transformaciones para el país, y especialmente para el agro. Esto significó borrar en forma significativa los cambios introducidos en la época anterior. Este nuevo proceso ha sido denominado Modernización Excluyente.

Esta modernización, en lo que atañe al sector agrícola presentó las siguientes características :

a. Plena incorporación de nuestra economía a los mercados mundiales a través de la producción de materias primas y productos de escaso valor agregado.

b. La puesta en práctica de la política sustentada en las ventajas comparativas que dio prioridad a la explotación frutícola y forestal (condiciones de suelos, climas y estacionalidad)

La organización de la producción en torno a la empresa agroexportadora que se yergue en el campo chileno como la unidad económica de mayor dinamismo. Su expresión máxima está representada en los complejos agroindustriales, que son el centro de una agricultura industrial, cuyos factores claves son los grandes volúmenes de capitales de inversión, uso de tecnología de punta y una moderna gestión empresarial.

d. La constitución de un nuevo Código Laboral, funcional a la actividad agroexportadora.

e. Una agricultura comercial que ha acentuado la temporalidad del trabajo agrícola, lo que se ha traducido en un tipo de trabajo precario, en el cual los actores más representativos son los temporeros.

Todo lo anterior ha incidido en una profunda alteración de los patrones tradicionales de asentamiento de la población rural. Estamos en presencia, en el campo de la Región Metropolitana de un paisaje muy distinto a los anteriores de los complejos agroindustriales, la vivienda tradicional con cerco o huerto ya no está en la presente estructura de las empresas agroexportadoras, las cuales realizan una valoración económica óptima de todo el espacio productivo, lo que conlleva a la expulsión de población residente en ellas. Al mismo tiempo, la especialización productiva ha modificado los requerimientos de fuerza laboral, dando énfasis al trabajo temporal. La demanda de mano de obra ha influído en el reordenamiento del asentamiento de la población, desarrollándose migraciones pendulares, semipermanentes y permanentes en diferentes direcciones. Lo anterior se refleja en una tendencia hacia la concentración espacial con un cambio en la funcionalidad de aldeas, pueblos y ciudades pequeñas. La población ahora tiene más bien un carácter rururbano por su vinculación laboral agrícola. En la figura $\mathrm{N}^{\circ} 4$, se puede constatar los lugares de residencia de los trabajadores temporeros. 
TABLA N³

Sector de residencia habitual de temporeros

\begin{tabular}{|l|l|}
\hline TIPO DE SECTOR & $\%$ \\
\hline & \\
\hline A. Agricultura campesina & 44.4 \\
- Pequeña propiedad y minifundio & \\
- Area de Reforma Agraria & 14.9 \\
- Comunidad Indígena & 1.8 \\
\hline B. Agricultura Empresarial & 7.0 \\
- Fundos, Hijuelas, Huertos, etc. & 7.0 \\
\hline C. Rural Urbanizado & 39.7 \\
- Pueblos y Aldeas & 39.7 \\
\hline D. Urbano & 12.9 \\
- Diversas ciudades & 9.8 \\
- Valparaíso - Santiago & 3.1 \\
\hline
\end{tabular}

Fuente : Gómez, Sergio y Jorge Echeñique "La agricultura chilena, las dos caras de la modernización" pág. 71.

Al interior de las áreas modernizadas es posible reconocer la presencia de la agricultura campesina, representada por los parceleros asignatarios, minifundistas e indígenas, los cuales han ido disminuyendo en número en forma paulatina. A pesar que este tipo de agricultura tenía manifestaciones mayores a través de diferentes formas ej. La medianería practicada por campesinos en predios hacendales y de mediana propiedad y que al desaparecer la primera, significó una reducción de ella en el mundo rural.

\section{El actual proceso de urbanización del campo metropolitano}

En los últimos años este proceso se ha acelerado significativamente y se ha expresado a través de dos modalidades: urbanización de elite (parcelas y condominios de agrado) y sub-urbanización.

\section{a) La urbanización de elite}

Esta modalidad de urbanización de las áreas rurales metropolitana está representada por las parcelas de agrado, que son unidades residenciales de manejo individual y los condominios, que reúnen a varias parcelas, que se administran en forma comunitaria y que a diferencia de la anterior se componen de predios de una menor dimensión. Sin embargo, ambos tipos representan a personas de elevados ingresos y que en su mayoría proceden del Gran Santiago.

Estas urbanizaciones son fácilmente distinguibles en el paisaje rural, porque conforman espacios característicos por el tipo de construcción de viviendas de elevada calidad, junto a una sofisticada infraestructura recreacional (piscinas, 
recintos deportivos, parques y jardines) y básica (sistemas de alcantarillado, agua potable, etc.).

Tanto las parcelas como los condominios de agrado se vinculan directamente con inmobiliarias y bancos que accionan el mercado de tierras sin que se observe una regulación del proceso (planificación). Las áreas privilegiadas han sido aquéllas de mayor accesibilidad y de mayor rentabilidad para la empresa comercial.

En la Comuna de Pirque (Fig. $N^{\circ} 1$ ) se ha podido constatar que las parcelas de agrado están presentes desde 1958, año en el que se constituyeron alrededor de unas 50 parcelas a lo largo de las riberas del río Mapocho, que comenzaron siendo residencias de fin de semana, pero que mantuvieron un uso del suelo agrícola, es decir el uso de los predios fue de carácter mixto (residencialagrícola). Durante el período posterior al año citado, la proliferación de estos predios fue limitada por el alto precio del suelo y la accesibilidad a Santiago. En relación a lo primero, esta situación se mantiene en la actualidad y el mejoramiento de la accesibilidad ha originado una residencia permanente.

La demanda de suelo urbano permaneció en esta comuna, relativamente estable en el período 1960-1980, pero esta situación cambia a partir del último año, en el que se incrementa la demanda de proyectos de loteos, que se manifestó en un incremento de construcciones de residencias rurales en un 65\%. Entre los años 1980-1990 se construyeron 1.100 viviendas particulares de alta calidad, localizadas en las áreas de loteos. Este aumento se realizó en conformidad a la ley 3.516 , la que permite la venta de superficies prediales mínimas de hasta $5.000 \mathrm{~m}^{2}$ en el ámbito rural.

Los principales focos de atracción para loteos se fueron situando paulatinamente en las localidades de El Cruceral, El Principal y Santa Rita, privilegiándose la cercanía al río Clarillo.

¿Cómo explicar las motivaciones que llevan a un determinado segmento de la población urbana a vivir en el campo? Sobre la base de las entrevistas realizadas en el área de estudio se obtuvieron conclusiones, de las cuales se pudo inferir que entre las razones está el deseo de vivir con un mayor contacto con la naturaleza para gozar del aire puro, con una vida más tranquila, lejos de la contaminación y la violencia de la gran ciudad.

La vida cotidiana de estos habitantes transcurre entre la ciudad, donde se encuentra el trabajo (ejecutivos y profesionales) y el campo, donde está la residencia. Por ello, estas parcelaciones se han constituído en enclaves residenciales de carácter urbano al interior del mundo rural, manteniendo en sí la dinámica propia de Santiago en relación a la segregación espacial. Su vinculación con el hábitat campesino circundante se reducen a la contratación de fuerza laboral mientras dura la construcción y luego, la contratación de servicios 
(cuidadores, jardineros y empleadas domésticas).

En otras áreas, se constata la influencia de otros factores, como es el caso de la Laguna de Aculeo (Comuna de Paine), donde el turismo se ha convertido en el principal agente de cambio de uso del suelo agrícola. Desde 1950, la atracción turística de la laguna, ha significado un fuerte estímulo a la construcción de residencias y campings, lo que ha generado un paisaje de balneario al igual que otros centros turísticos, del país. Surge, entonces, una verdadera ciudad veraniega que continúa expandiéndose hacia los cerros vecinos, en donde la panorámica de la laguna se ha convertido en una mercancía del negocio inmobiliario.

Resulta interesante destacar en este último caso, la percepción que tiene el campesino acerca de estos procesos. Primeramente, se observa una percepción negativa de los campesinos del lugar frente al avance de la urbanización y corresponde a aquéllos que vendieron sus tierras a muy bajo precio, dejando de ser propietarios. Otros campesinos lo perciben, en cambio, en forma positiva puesto que se han incorporado a la fuerza laboral que demanda esta nueva forma urbana, o bien se han convertido en trabajadores informales a través del pequeño comercio. Recientemente, se verifica que algunos campesinos han realizado un aprendizaje en las transacciones comerciales de tierras, puesto que exigen altos precios por sus predios, que aún poseen ( 3 millones de pesos la hectárea de secano, año 1995).

b) La sub-urbanización

Se entiende por sub-urbanización al proceso que afecta al hábitat rural y periurbano y a la estructura espacial que se manifiesta en el surgimiento de conglomerados poblacionales que tienen una precaria calidad de vida, con una deficiente infraestructura social básica.

Este proceso se localiza de preferencia en antiguas localidades campesinas y en los campamentos espontáneos rurales.

1. Localidades campesinas. Aquí se concentran diferentes categorías de campesinos: minifundistas, parceleros o medieros. Los más numerosos son los parceleros o campesinos asignatarios, como se les llama. Ellos obtuvieron sus tierras, a través del proceso que llevó a cabo la Corporación de Reforma Agraria (CORA), entre los años 1975-1978. Las tierras asignadas fueron parcelas agrícolas con un tamaño promedio de 8HRB (hectárea de riego básico)

La mayoría de estos propietarios vendieron sus tierras, debido a la falta de capital de inversión, al no cumplimiento de sus deudas con CORA y a la falta de apoyo del Estado. El nuevo modelo económico en el que estaban inmersos no fue propicio a la persistencia de esta agricultura campesina. Se inició así una fase de traspaso de tierras campesinas a medianos empresarios agrícolas y 
posteriormente, a inmobiliarias. En el caso de la Comuna de San Bernardo, un $80 \%$ de los asignatarios enajenaron sus predios.

Los campesinos que han vendido sus parcelas, aún mantienen el sitio (5.000 $\mathrm{m}^{2}$ ), donde está su vivienda. El problema interno que les afecta en la subdivisión de la propiedad por efecto de la herencia. En muchos sitios se detectaron más de 2 viviendas familiares, que corresponden a los hijos que han constituído sus hogares y residen allí porque se han transformado en "pobladores rurales sin casa". Estas viviendas adicionales a la paterna (familias allegadas) conforman verdaderos tugurios debido a la gran presión de población. Por lo demás, también se constata la inexistencia de explotaciones agropecuarias que signifique un excedente económico, limitándose el área que resta de los sitios a pequeños huertos de subsistencia y cría de aves.

La fisonomía que presentan estas localidades campesinas es muy característica, situadas a lo largo de los caminos forman verdaderas conurbaciones rurales ej.: sitios campesinos de San Adolfo y San Jorge en la Comuna de San Bernardo.

2. Campamentos Rurales. Estos campamentos corresponden a situaciones distintas de las anteriores, pero forman parte de una misma realidad. Se trata en todo caso de pobladores rurales que carecen de vivienda y por ello se instalan en forma espontánea en áreas, generalmente de propiedad fiscal. A esto se agrega además, otro carácter, ser áreas de riesgos (lecho de los ríos, cercanía de canales de riego, líneas férreas, carreteras, etc.).

Los autores de este trabajo han realizado estudios en dos campamentos : Los Morros (Comuna de San Bernardo) y Los Guindos (Comuna de Buin).

El primero de ellos está situado en la cuenca del Río Maipo, sector norte Camino-Puente Los Morros (Fig. $N^{\circ}$ 2). Este asentamiento informal es de larga data, ya que se originó hacia 1930. El sustento económico de los primeros que allí se establecieron y construyeron su hogar fue la extracción de áridos (arena y ripio) para la industria de la construcción. Esta actividad aún se mantiene vigente y constituye la fuente principal del ingreso familiar.

Una de las características del campamento era su estabilidad en el número de viviendas y por ende, de hogares, porque a la salida o abandono de una familia, debido a la obtención de una vivienda localizada en áreas de mejor calidad de vida, se registra la llegada de otro grupo familiar. Este fenómeno de movilidad es completamente irregular en el tiempo y en el espacio.

La pobreza constituía el rasgo distintivo de estos pobladores, ya que ella no sólo se debía a sus bajos ingresos económicos, sino que, a la paupérrima calidad de la infraestructura social básica, debido a que sólo poseían electricidad mientras que el agua les era suministrada a través de camiones aljibes por el municipio. 
Su fuente laboral continuaba siendo la explotación del lecho del río, la que se complementaba con actividades temporeras en la fruticultura de exportación del área agrícola circundante.

El número de familias era alrededor de 40, (población aproximada de 70 personas, con muchos niños). Se destacaba un aspecto muy importante; tenían sus organizaciones sociales (Junta de Vecinos y Club-Deportivo). Fue interesante constatar que estos pobladores no percibían los riesgos a los cuales estaban expuestos, a pesar de que la línea de más alto caudal del río podía llegar a $10 \mathrm{~m}$. de sus hogares. FIGURA N²

\section{Campamento Los Guindo (Fig. №3)}

Se localiza al noreste de la ciudad de Buín, entre la Ruta Panamericana $N^{0} 5$ sur y la vía del ferrocarril sur, por un largo aproximado de 1.400 m. en dirección norte-sur y por un ancho máximo de 60 y un mínimo de 30 metros. Existían en total 123 sitios ocupados por 200 familias, con 438 personas (año 1995).

El origen de este asentamiento se debió a que un agricultor propietario instaló allí familias campesinas con el fin de resguardar los límites de su hacienda. Este hecho sucedió a principio de la década de los 50.

Durante su existencia ha sufrido 2 erradicaciones de toda la población, hacia diversas áreas circundantes, produciéndose el fenómeno del retorno de una parte de dichas, familias que han regresado por motivos laborales e incapacidad económica para enfrentar el pago de las nuevas viviendas.

Respecto a la infraestructura social básica, ésta se caracterizaba por ser mínima y sólo en los últimos años (anteriores a la investigación) se les incorporó la luz y el agua, cuyo pago era comunitario.

Un rasgo distintivo de este campamento era el constante arribo e incorporación de nuevos pobladores. El 47\% había llegado con posterioridad al año 90 y los más antiguos, sólo se remontaban al año 1978, lo que coincidía con los períodos de mayor expansión de la actividad frutícola de la Comuna de Buín, la más importante área agroexportadora de la Región Metropolitana. Por esta razón, el campamento era un foco de atracción de migrantes. Aproximadamente, el $60 \%$ de la población provenía de la provincia del Maipo, un $10 \%$ de la ciudad de Santiago y alrededor de un $20 \%$ de la VII y VIII regiones del país.

Las aspiraciones de la mayor parte de esta población se concentran en una vivienda estable en un área de menor riesgo. Pese a ello manifestaban un fuerte arraigo al lugar donde toda su vida había transcurrido y porque de una manera u otra consideraban que allí tenían asegurado el empleo.

Observación: Este campamento, iba a ser erradicado por el Municipio de Buín 
1995.

\section{Conclusiones}

La urbanización del campo que se observa en las áreas de estudio de las comunas de San Bernardo, Pirque, Paine y Buin en la Región Metropolitana, presenta características antagónicas, pero concomitantes entre sí. La urbanización de elite y la sub-urbanización. Estas se han dinamizado en los últimos años, con la consiguiente agudización del ya deteriorado hábitat campesino, junto a la pérdida de suelos agrícolas. En esencia, este proceso conlleva implícitas contradicciones sociales que se traducen en fenómenos de segregación social.

Se pueden advertir diferenciaciones territoriales en esta dinámica, producto de factores que imprimen un sello particular a cada área. Así por ejemplo, en Pirque hay una transformación paulatina de una comuna agrícola - rural a una comuna agroexportadora - residencial, lo que implica un impacto negativo en la economía y hábitat campesino. En este último caso, se conjugan las agroindustrias y parcelas de agrado para constreñir el ámbito productivo, laboral y cultural del campesino. En cambio, en el sector de la Laguna de Aculeo, aunque se repiten los efectos negativos sobre la vida y quehacer campesinos, el turismo es el agente dinamizador fundamental.

Por otra parte, en todas estas situaciones, las inmobiliarias con apoyo financiero, desde la gran ciudad, controlan las rentas de la tierra, disputando estas ganancias a los propietarios del suelo, a través de un proceso no regulado y más bien espontáneo, que es inherente a la economía de mercado. Esto se asemeja al período que se dio en Santiago durante el "boom" inmobiliario de la década de los 80 . Aunque, la normativa vigente permite la subdivisión hasta $5.000 \mathrm{~m}^{2}$, de hecho se están produciendo alteraciones a esta norma legal, puesto que mediante el subterfugio de los condominios de elite se está realizando una fragmentación mayor al transarse residencias entre 1.000 y $2.000 \mathrm{~m}^{2}$.

Por último, es importante señalar que los habitantes tradicionales de este medio, es decir los campesinos, tienen mayoritariamente una visión negativa de este proceso que está alterando la estructura agraria y sus formas de vida. Para otros, este cambio es visto positivamente, puesto que perciben mayores perspectivas laborales.

\section{Bibliografía}

1. Armijo Gladys, Caviedes Héctor 1996, La actual urbanización del mundo Rural de la Región Metropolitana (Area Sur) y sus efectos sobre el hábitat campesino. Anales XVII Congreso Nacional de Geografía, La Serena, Chile.

2. CEPAL, 1995, Alojar el desarrollo una tarea para los asentamientos humanos.CIREN-CORFO, 1992, Avance urbano y pérdida de suelos agrícolas 
en el Gran Santiago entre 1970-1991.

3. CIREN-CORFO, Avance urbano y pérdida de suelos agrícolas en el Gran Santiago entre 1970-191, 1992

4. Cruz, María Elena 1987, Chile, clases sociales y cambio agrario. Grupo de Investigaciones agrarias GIA.

5. Echenique Jorge y Sergio Gómez 1988, La agricultura chliena. Las dos caras de la modernización, agraria. FLACSO.

6. Gutíerrez V. Alejo, 1995, Migraciones y situación laboral en Los Guindos. Comuna de Buin. Magister en Geografía, Universidad de Chile (no publicado)

7. Hurtado, Carlos 1966, Concentración de población y desarrollo económico. El caso chileno. Instituto de Economía, Universidad de Chile.

8. Instituto Nacional de Estadísticas INE Censos de Población Chile, 1987-1992.

9.Uribe, Graciela 1996, Geografía Política. Verdades y falasias de fin de mileno. México. 